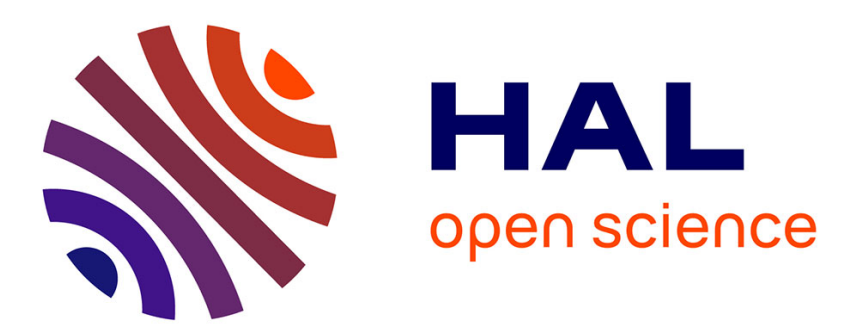

\title{
The constraint functions: an attempt to evaluate the constraint rate inside structures that undergo ordered magnetic frustration
}

P. Lacorre

\section{- To cite this version:}

P. Lacorre. The constraint functions: an attempt to evaluate the constraint rate inside structures that undergo ordered magnetic frustration. Journal of Physics C: Solid State Physics, 1987, 20 (29), pp.L775-L781. 10.1088/0022-3719/20/29/004 . hal-02183468

\section{HAL Id: hal-02183468 \\ https://hal.science/hal-02183468}

Submitted on 16 Jul 2019

HAL is a multi-disciplinary open access archive for the deposit and dissemination of scientific research documents, whether they are published or not. The documents may come from teaching and research institutions in France or abroad, or from public or private research centers.
L'archive ouverte pluridisciplinaire HAL, est destinée au dépôt et à la diffusion de documents scientifiques de niveau recherche, publiés ou non, émanant des établissements d'enseignement et de recherche français ou étrangers, des laboratoires publics ou privés. 


\title{
The constraint functions: an attempt to evaluate the constraint rate inside structures that undergo ordered magnetic frustration
}

\author{
P. Lacorre \\ Institut Laue-Langevin, 156X Avenue des Martyrs, 38042 Grenoble Cédex, France and \\ Laboratoire des Fluorures, UA449 Université du Maine, Route de Laval, 72017 Le Mans \\ Cédex, France
}

\begin{abstract}
We propose a simple way to determine the constraint rate in ordered frustrated magnetic structures, based upon the calculation of the reduced energy of the system. The notion of a constraint function is illustrated using the example of the symmetrical triangular platelet, for which the evolution of constraint with the coupling constants ratio is studied. The constraint rates of some classical structures and real compounds estimated by this method are presented.
\end{abstract}

The notion of frustration has been introduced by $\mathrm{G}$ Toulouse to explain the spin glass behaviour of some compounds [1]. The frustration can be defined as the inability of a given interacting system to satisfy simultaneously all the interactions that it undergoes. This notion was first developed by considering a lattice of square platelets of Ising spins. Toulouse defined the frustration function $\Phi_{k}$ of a platelet as the product of the signs of the interactions along the contour of this platelet [1]. If $\Phi_{k}$ is negative, the platelet is said to be frustrated.

This concept of frustration, which allows disordered magnetic systems to be described satisfactorily, may be generalised to ordered systems of $X Y$ or Heisenberg spins, for which one can adopt a similar definition. A quantitative definition of the terms involved is then necessary: conditions for a ferromagnetic interaction will be satisfied only if the arrangement of interacting spins is strictly parallel, and for an antiferromagnetic one only if the arrangement of spins is strictly antiparallel. From this definition, a nonfrustrated magnetic structure is necessarily collinear; the reverse is not always true.

In this Letter, we will consider the ordered magnetic frustration of isotropic or weakly anisotropic systems, or more generally of systems for which the anistropy does not add further constraint to that intrinsic to exchange. We use for the exchange Hamiltonian the general form of two-spin coupling expressed as

$$
\mathscr{H}=-\sum_{\langle i, j\rangle} J_{i j} S_{i} \cdot S_{j}
$$

where $S_{i}$ and $S_{j}$ are Heisenberg spins (although what follows can be generalised to multispin coupling). 
As the ground state of a system is that of lowest energy, it is not the sign of the interactions, nor even their strength, but rather their energy that will determine the spin arrangement. Thus we may propose, for a pair interaction, what we call its basis energy $e_{b}$, which would represent the energy of this pair interaction if it were not frustrated: $e_{b}=-\left|J_{i j}\right|\left|S_{i}\right|\left|S_{j}\right|$. In a frustrated platelet, the more negative the basis energy of an interaction is, the more the configuration of spins connected by this interaction will tend toward collinearity. If two interactions have identical basis energies, the angle between the directions of corresponding spins will be identical, whatever the signs of these interactions.

The energy of a system consisting of $n$ interactions will always be greater than or equal to its basis energy; it is expressed as

$$
E_{\mathrm{b}}=\sum_{k=1}^{n} e_{\mathrm{b}}(k)
$$

The larger the departure from the basis energy, the more frustrated the structure will be. Thus the exchange energy seems a good gauge of the constraint rate of a frustrated structure, but is not really manageable enough for our purposes because it is an extensive parameter. We prefer to use the reduced energy of the system, defined by the ratio of its energy to its basis energy, which we call a 'constraint function'. For a system involving $n$ interactions, the constraint function is thus expressed as

$$
F_{\mathrm{c}}=-E / E_{\mathrm{b}}=-\sum_{k=1}^{n} J_{k} S_{k 1} \cdot S_{k 2} / \sum_{k=1}^{n}\left|J_{k}\right|\left|S_{k 1}\right|\left|S_{k 2}\right|
$$

$F_{\mathrm{c}}$ lies in the range -1 (non-frustrated system) to +1 (fully frustrated). $F_{\mathrm{c}}$ may be calculated for a single interaction, a group of interations, a platelet, a magnetic cluster or a periodic magnetic structure and allows, without rescaling, direct comparison of the frustration rates of these various magnetic arrangements.

The constraint rate $R_{\mathrm{c}}$ may be defined from the constraint function by $R_{\mathrm{c}}=\frac{1}{2}\left(1+F_{\mathrm{c}}\right)$. $R_{\mathrm{c}}$ lies between 0 (no constraint) and 1 (maximum constraint). One can also define a constraint angle $\theta_{\mathfrak{c}}$, which corresponds to the 'mean angle of departure from collinearity', by the relation $\theta_{\mathrm{c}}=\cos ^{-1}\left(-F_{\mathrm{c}}\right)$. Angle $\theta_{\mathrm{c}}$ is equal to zero for a non-frustrated structure and increases with the constraint rate.

The most common and widely studied system undergoing ordered (or disordered) magnetic frustration is the triangular lattice with antiferromagnetic interactions $\left(\Phi_{k}=\right.$ $-1)$. Practically, this special geometry is encountered in some peculiar structural types (see for instance [2]) and has recently been systematically investigated in fluorides of $3 \mathrm{~d}$ transition elements ([3] and references therein). In these compounds, the basal antiferromagnetic interaction is often provided by a super-exchange path via cornersharing octahedra. With such a configuration, the balanced coupling constants lead to a $120^{\circ}$ star arrangement of spins inside triangular platelets, in some cases with a noticeable departure from the ideal configuration (see for instance [3]). In other more specific cases, one of the three interactions of the platelet takes place via a $90^{\circ}$ super-exchange path, the two others remaining of $180^{\circ}$ type. Such a conformation occurs for instance in two structural configurations (presented in figure $1(a)$ ), which are found in the rutile structural type and in compounds like $\mathrm{BaMnFe}_{7}[4]$. The sign of the $90^{\circ}$ super-exchange integral depends essentially on the electronic configuration of the magnetic cations and may be either ferromagnetic or antiferromagnetic. However such a system can be described as a symmetrical triangular platelet with two types of interactions, one called 
(a)
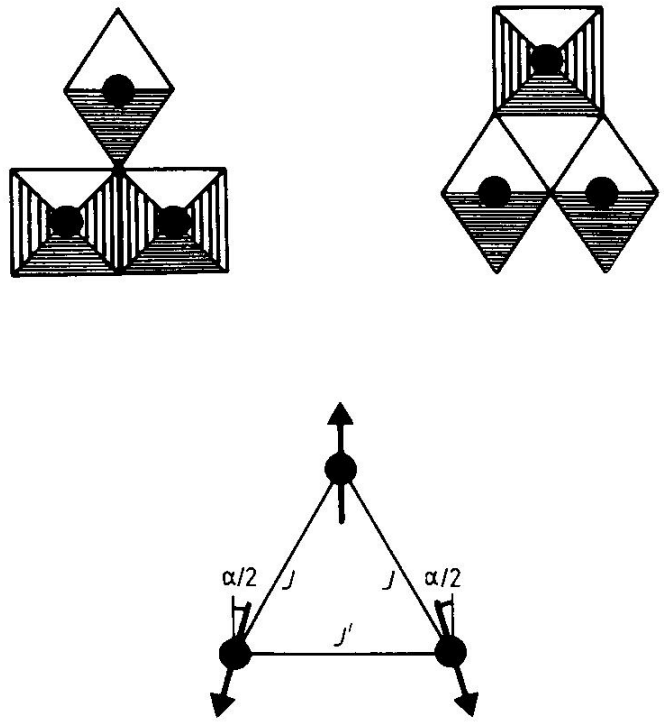

Figure 1. Two possible arrangements ( $a$ ) of coordination polyhedra (octahedra) of magnetic cations (as encountered for instance in the rutile structure) which form a symmetrical triangular platelet $(b)$.

$J^{\prime}$ and two $J$ (figure $1(b)$ ). We have undertaken the study of this system for $X Y$ spins, keeping the coupling constant $J$ (which is the general case for a $180^{\circ}$ super-exchange path) antiferromagnetic and allowing $J^{\prime}$ to vary from positive to negative values. For the sake of simplicity, the spin amplitude has been set to 1 , the varying parameter being $X=$ $J^{\prime} / J$. We shall only consider the isotropic case. If $\alpha$ denotes the canting angle between the spins connected by the interaction $J^{\prime}$, the energy of the system can be expressed as $E=J(2 \cos \alpha / 2-X \cos \alpha)$. The basis energy of the platelet, as defined as above, is given by $E_{\mathrm{b}}=-2|J|-\left|J^{\prime}\right|$. Since $J$ is negative, $|J|=-J$ and $E_{\mathrm{b}}=J(2+|X|)$. We can see that the expression for the basis energy depends on the signs of the interactions.

Besides the total constraint function of the platelet $F_{\mathrm{c}}=-E / E_{\mathrm{b}}$, we can calculate the local constraint function corresponding to each of the interactions. When applied to a single interaction $J_{k}$, equation (1) reduces to the simple expression $F_{\mathrm{c}}(k)=$ $-\operatorname{sgn}\left(J_{k}\right) \cos \left(S_{k 1}, S_{k 2}\right)$.

Table 1 gives the results of calculations concerning the ground-state configuration of the symmetrical triangular platelet as a function of $X$. It gives the evolution of the canting angle $\alpha$, the energy $E$, the frustration function $\Phi_{k}$ according to Toulouse, the total

Table 1. The canting angle $\alpha$, energy $E$ and constraint functions $\left(F_{\mathrm{c}}, f_{\mathrm{c}}, f_{\mathrm{c}}^{\prime}\right)$ inside the symmetrical triangular platelet as functions of the coupling constants ratio $X$.

\begin{tabular}{lllll}
\hline$X$ & $-\infty$ & 0 & $\frac{1}{2}$ & $+\infty$ \\
\hline$\alpha(X)$ & 0 & 0 & $2 \cos ^{-1}(1 / 2 X)$ \\
$E(X)$ & $J(2-X)$ & $J(2-X)$ & $J(X+1 / 2 X)$ \\
$\Phi_{k}(X)$ & +1 & -1 & -1 & $-\left(2 X^{2}+1\right) / 2 X(2+X)$ \\
$F_{c}(X)$ & -1 & $(X-2) /(X+2)$ & $1 / 2 X^{2}-1$ \\
$f_{c}^{\prime}(X)$ & -1 & +1 & $-1 / 2 X$ \\
$f_{\mathrm{c}}(X)$ & -1 & -1 & \\
\hline
\end{tabular}


constraint function $F_{\mathrm{c}}$ and the local constraint functions $f_{\mathrm{c}}$ and $f_{\mathrm{c}}^{\prime}$ relative to interactions $J$ and $J^{\prime}$ respectively. Figure 2 illustrates the previous analytical expressions, and the good agreement with the results of Monte Carlo simulations. Figure 3 shows the evolution of the total and local constraint functions of the platelet.

From these results, one can draw the following conclusions.

(i) The occurrence of magnetic frustration above $X=0\left(\Phi_{k}=-1\right.$ for $\left.X>0\right)$ has no direct effect on the canting angle or on the energy of the system, which both keep their linear dependence on $X$ through $X=0$.

(ii) The collinear configuration $(\alpha=0)$ is stable until $X=\frac{1}{2}\left(J^{\prime}=J / 2\right)$. In the range $0<X \leqslant 0.5$ the stable arrangement of spins connected by the interaction $J^{\prime}$ is parallel, while $J^{\prime}$ is antiferromagnetic.

$(a)$

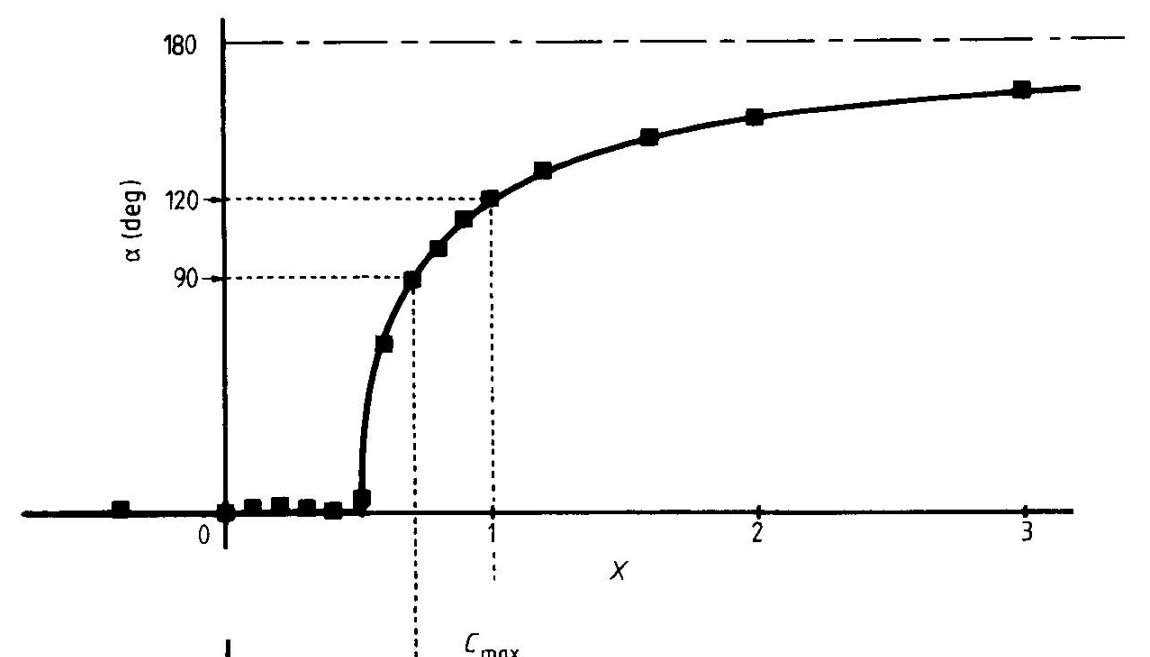

(b)

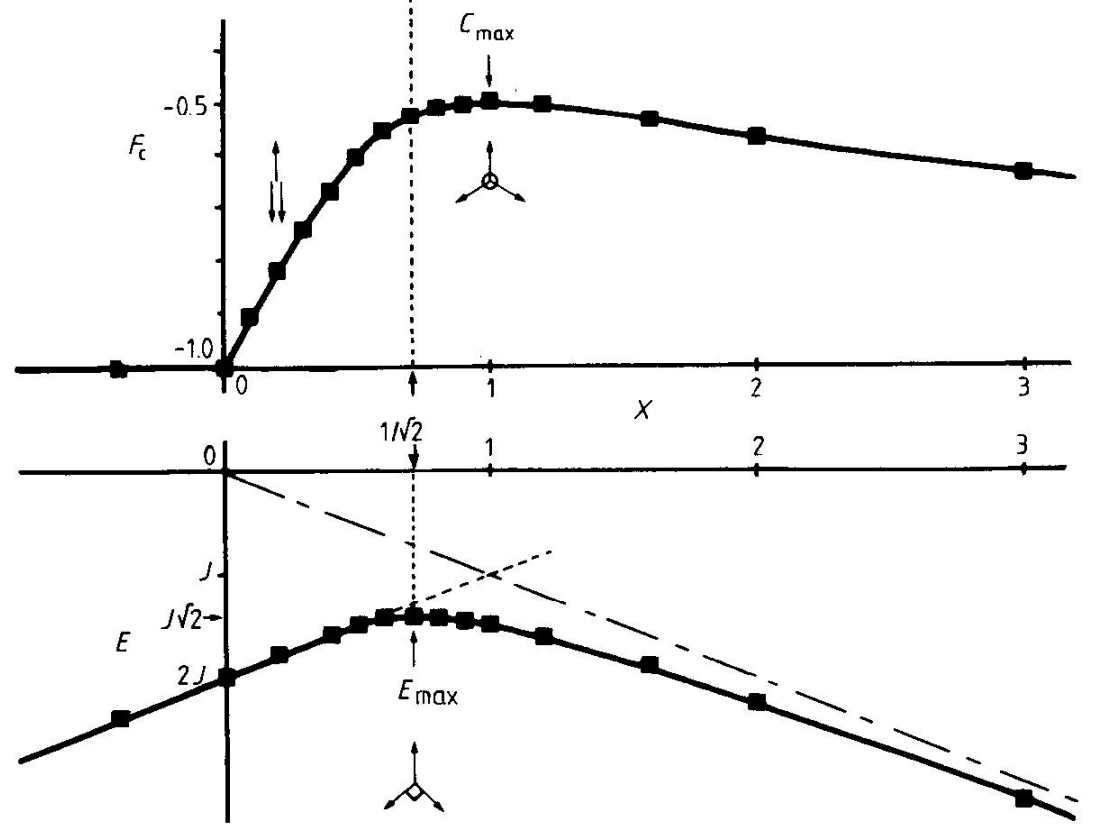

Figure 2. (a) Canting angle; (b) constraint function and (c) energy of the symmetrical triangular platelet versus the coupling constants ratio $X=J^{\prime} / J$. Full curves refer to analytical results and full squares to Monte Carlo simulation results. $C_{\max }$ is the point of maximum constraint, $E_{\max }$ the point of maximum energy. 
(iii) Unlike the canting angle and the energy, the total constraint function becomes different from -1 straight away from $X=0$, indicating the onset and strengthening of the constraint inside the platelet.

(iv) Both the energy and the constraint function pass through a maximum, but not for the same value of $X$. The maximum value of the energy occurs at $X=\frac{1}{2} \sqrt{2}$, corresponding to a canting angle of $90^{\circ}$, whereas the maximum value of the constraint function corresponds to $X=1$, i.e. $J^{\prime}=J$ and $\alpha=120^{\circ}$, the classical ground-state configuration of the triangular platelet with antiferromagnetic interactions [5].

(v) When $X$ tends towards infinity $(J>0)$, the canting angle tends towards $180^{\circ}$ and simultaneously the constraint rate decreases. For $J=0$, the system is non-frustrated $\left(F_{\mathrm{c}}=-1\right)$.

(vi) Concerning the local constraints (figure 3), it can be seen that from $X=0$ to $X=\frac{1}{2}$ the only frustrated interaction is $J^{\prime}, J$ remaining unfrustrated. The situation is progressively reversed as $X$ increases. The local constraint function $f_{\mathrm{c}}^{\prime}$ undergoes a discontinuity at $X=0$, which corresponds to the discontinuity in the frustration function $\Phi_{k}$. The maximum of energy corresponds to the point where $f_{c}^{\prime}=0\left(X=\frac{1}{2} \sqrt{ } 2\right)$, while the maximum of the constraint function occurs when $f_{\mathrm{c}}=f_{\mathrm{c}}^{\prime}=F_{\mathrm{c}}=-0.5(X=1)$.

As illustrated here, the constraint functions seem to offer a more accurate way to evalute and quantify the constraint rate of any magnetically ordered system than the frustration function. For instance in the above system the collinear arrangement is stable up to a strong constraint rate $\left(F_{\mathrm{c}}=-0.6 \Leftrightarrow R_{\mathrm{c}}=20 \%\right)$ which corresponds to $80 \%$ of the maximum constraint rate occurring in this system $\left(F_{\mathrm{c}}=-0.5 \Leftrightarrow R_{\mathrm{c}}=25 \%\right.$ for all interactions set equal).

The calculation of the constraint function has been incorporated into the computer program MCMAG, designed to simulate magnetic models and real magnetic structures from the coupling constant values [6]. The constraint rates of some structural types with frustrating topologies and of some compounds have thus been estimated. The results are presented in table 2 .

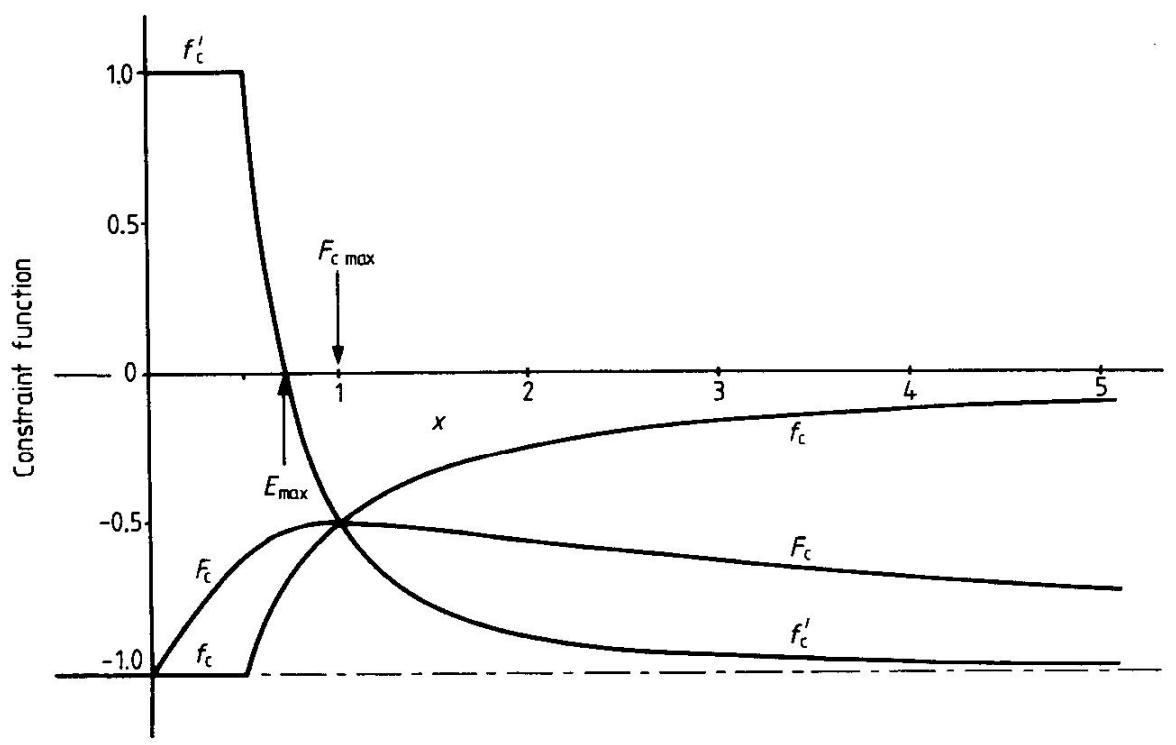

Figure 3. Total $\left(F_{c}\right)$ and local $\left(f_{c}, f_{c}^{\prime}\right)$ constraint functions inside the symmetrical triangular platelet as a function of $X$. 
Table 2. Evaluation of the magnetic constraint in a few frustrating structures or topologies.

\begin{tabular}{|c|c|c|c|}
\hline Structures $\dagger$ & $F_{\mathrm{c}}$ & $R_{\mathrm{c}}(\%)$ & $\theta_{\mathrm{c}}(\mathrm{deg})$ \\
\hline \multicolumn{4}{|l|}{$\begin{array}{l}\text { Ferromagnets, } \\
\text { unfrustrated antiferromagnets, }\end{array}$} \\
\hline$\left(\mathrm{K}_{2} \mathrm{NiF}_{4}, \mathrm{Rh}-\mathrm{FeF}_{3}\right.$ etc $)$ & -1 & $\mathbf{0}$ & 0 \\
\hline & -0.967 & 1.7 & 14.8 \\
\hline AF tetragonal bronze $\dagger$ & -0.800 & 10.0 & 36.9 \\
\hline Column of $\mathrm{KCrF}_{4}$ & -0.800 & 10.0 & 36.9 \\
\hline AF dodecahedral cluster $\dagger$ & -0.745 & 12.8 & 41.8 \\
\hline \multicolumn{4}{|l|}{ Frustrated square platelet } \\
\hline$(+++-)$ & -0.707 & 14.7 & 45 \\
\hline \multicolumn{4}{|l|}{ AF hexagonal bronze $\uparrow$} \\
\hline$\left(\right.$ HTB-FeF $\left.{ }_{3}\right)$ & -0.667 & 16.67 & 48.2 \\
\hline Weberite AF $\dagger$ & -0.600 & 20 & 53.1 \\
\hline Helimagnet $\beta-\mathrm{MnO}_{2}$ & -0.552 & 22.3 & 56.5 \\
\hline AF triangular platelet $\dagger$ & -0.500 & 25 & 60 \\
\hline $\mathrm{MnFeF}_{5} \cdot 2 \mathrm{H}_{2} \mathrm{O}$ & -0.500 & 25 & 60 \\
\hline AF icosahedral cluster $\dagger$ & -0.447 & 27.7 & 63.45 \\
\hline \multicolumn{4}{|l|}{ Pyroclore AF $\dagger$} \\
\hline$\left(\mathrm{Pyr}-\mathrm{FeF}_{3}\right)$ & -0.333 & 33.33 & 70.53 \\
\hline \multirow{3}{*}{$\begin{array}{l}\text { Interaction } \mathrm{Cr}_{1}-\mathrm{Cr}_{2} \text { in } \mathrm{KCrF}_{4} \\
\text { Interaction } \mathrm{Ni}_{1}-\mathrm{Ni}_{1} \text { in } \mathrm{Ba}_{2} \mathrm{Ni}_{3} \mathrm{~F}_{10} \\
\text { Saturated antiferromagnets } \\
\text { under high magnetic field }\end{array}$} & +0.292 & 64.6 & 107 \\
\hline & +1 & 100 & 180 \\
\hline & +1 & 100 & 180 \\
\hline
\end{tabular}

$\dagger \mathrm{AF}$ : interactions, between nearest neighbours only, are taken all equal and antiferromagnetic. References to the structural arrangements and magnetic structures can be found in [6].

The bottom part of the table concerns so-called induced frustration (for an external magnetic field) and/or local constraints (one peculiar interaction inside a frustrated compound, a case that is also relevant to induced frustration). The upper part of the table is connected with self-frustration. It presents the calculated internal magnetic constraints of a few structural types and real compounds, classified by increasing constraint rate. Among the compounds studied, $\mathrm{FeF}_{3}$ is a particularly interesting example because of its polymorphism [7-9]. Each of the three forms of $\mathrm{FeF}_{3}$ exhibits a different magnetic constraint rate due to its topology, with no constraint for the rhombohedral form (Rh perovskite type), an intermediate constraint for the hexagonal tungstenbronze-like form (HTB, [7]) and a strong constraint for the pyrochlore form (Pyr, $[8,9]$ ). In the frustrated forms of this family, one observes a drastic reduction of the saturated magnetic moments [9]. Figure 4 shows the value of this moment measured for all three forms versus the constraint rate of each structure (al! interactions are considered between nearest neighbours only, AF, and of the same amplitude, which is a reasonable approximation). The value exhibits a roughly linear decrease, which strengthens the assumption that the reduction of the magnetic moment is connected to the frustration rate. A decrease of the ordering temperature is also observed in these compounds [9].

We have thus presented a simple method for weighting the constraint rate inside compounds that undergo ordered magnetic frustration. The notion of the constraint function could easily be extended to frustrated anisotropic systems where the magnetic constraint arises from both anisotropy and topology, and, with little adjustment, to 


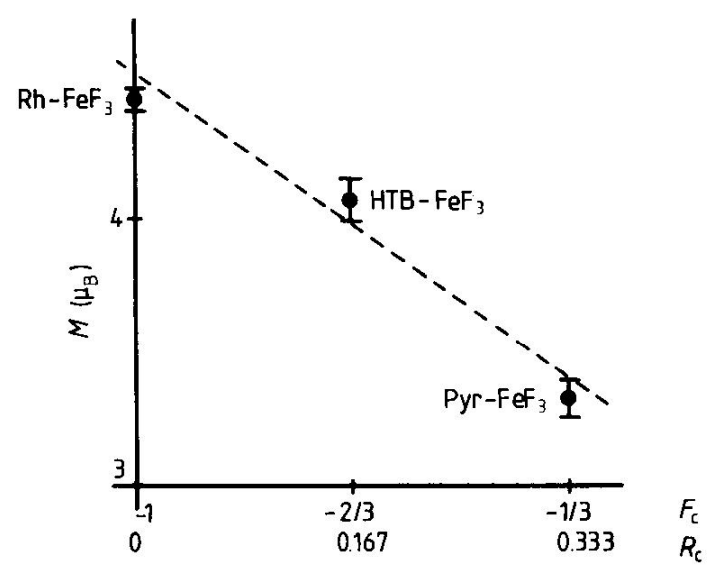

Figure 4. Saturated magnetic moments observed in the three crystalline forms of $\mathrm{FeF}_{3}$ [9] versus the approximate constraint rates of their magnetic structures.

systems that present a spin glass behaviour. Extension to non-magnetic systems ought to be possible. The constraint rate could be connected to particular physical properties of frustrated systems.

The author is very much indebted to Professor G Ferey and Dr J Pannetier for helpful suggestions and critical readings of the manuscript.

\section{References}

[1] Toulouse G 1977 Commun. Phys. 2115

[2] Zandbergen H W 1981 J. Solid State Chem. 37308

[3] Lacorre P, Leblanc M, Pannetier J and Ferey G 1987 J. Magn. Magn. Mater. 66219

[4] Holler H and Babel D 1981 J. Solid State Chem. 39345

[5] Marland L G and Betts D D 1979 Phys. Rev. Lett. 431618

[6] Lacorre P and Pannetier J J. Magn. Magn. Mater. submitted

[7] Leblanc M, De Pape R, Ferey G and Pannetier J 1986 Solid State Commun. 58171

[8] De Pape R and Ferey G 1986 Mater. Res. Bull. 21971

[9] Ferey G, De Pape R, Leblanc M and Pannetier J 1986 Rev. Chim. Miner. 23474 